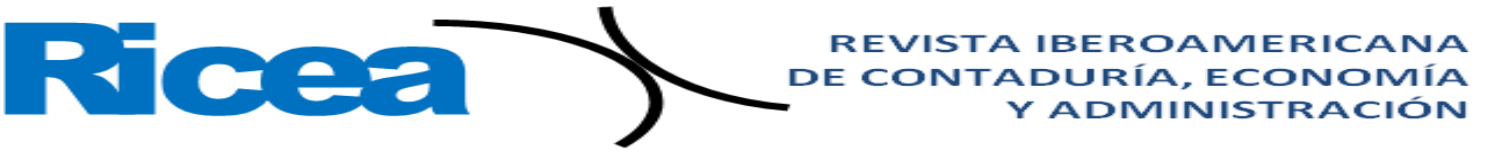

Artículos Científicos

\title{
Elementos mercadológicos necesarios para la subsistencia de la microempresa comercial, municipio de Chiautempan, Tlaxcala
}

\begin{abstract}
Market elements necessary for the subsistence of the commercial microenterprise, Municipality of Chiautempan, Tlaxcala
\end{abstract}

Elementos de mercado necessários para a subsistência da microempresa comercial, município de Chiautempan, Tlaxcala

Raúl Alonso Lozada

Universidad Tecnológica de Tlaxcala, México raul.alonso@uttlaxcala.edu.mx https://orcid.org/0000-0002-2435-7501

Armando Villanueva Meléndez

Universidad Tecnológica de Tlaxcala, México armandonueva@uttlaxcala.edu.mx https://orcid.org/0000-0001-9132-9386

Adriana Montiel García

Universidad Tecnológica de Tlaxcala, México adriana.montiel@uttlaxcala.edu.mx https://orcid.org/0000-0002-6984-9737

Ma. Luisa Espinoza Águila

Universidad Tecnológica de Tlaxcala, México mariluea@uttlaxcala.edu.mx https://orcid.org/0000-0002-5448-1116

Julissa Tizapantzi Sánchez

Universidad Tecnológica de Tlaxcala, México

julissats@uttlaxcala.edu.mx https://orcid.org/0000-0002-9828-8844 


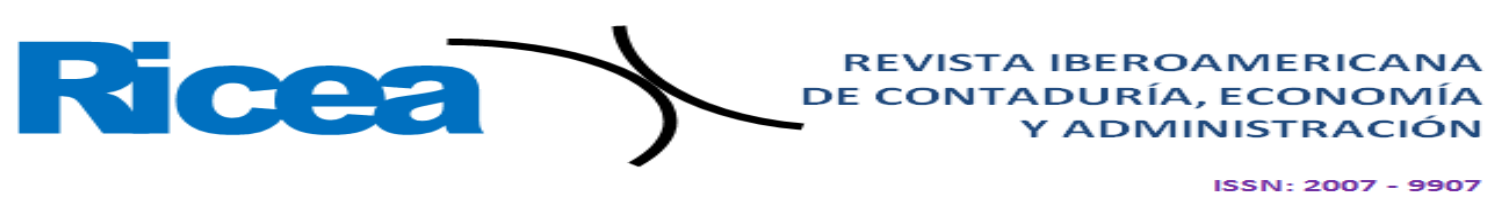

Otra parte esencial que describe a las microempresas del país es el tipo de producción que manejan. Se presume que la importancia de producir se enfoca más en la cantidad que en la calidad, a lo cual se suma que escasamente se manejan esquemas de continua innovación y crecimiento en la fuerza de ventas.

De acuerdo con el Sistema de Información Empresarial de México (SIEM), 92.3\% de las empresas no superan los diez empleados, por lo que existen alrededor de 603368 microempresas en todo el país. Este dato invita a pensar que la mejor forma de estimular el crecimiento económico es mediante los fenómenos que propician el crecimiento de este tipo de empresas, e identificando las deficiencias que limitan su periodo de vida.

Sin embargo, vale acotar que el presente trabajo de investigación busca demostrar que más allá de impulsar el crecimiento de un mayor número de microempresas, lo que en realidad se necesita es establecer estrategias que permitan elevar la calidad y productividad de las existentes, para lo cual es esencial lograr un adecuado manejo administrativo y, en especial, mercadológico.

Ambos temas son necesarios en la actividad microempresarial. Las instancias responsables de aplicar programas de estímulo a este sector pocas veces las han hecho válidas. Conocer la manera cómo se administra o se establece un plan de mercadotecnia es vital, ya que esto puede repercutir en el aumento o disminución de su tiempo de vida.

\section{La microempresa}

La mayoría de las economías de los países en vías de desarrollo dependen directa o indirectamente de las empresas. Derivado de la gran cantidad de empleos y recursos económicos que realizan en sus entornos, en el caso México las micro, pequeñas y medianas empresas son las dominantes, de ahí que sea necesario definir sus características primordiales.

A principios de la década de 1980, la investigación sobre las microempresas era prácticamente nula. Sin embargo, empezó a tomar interés a partir de que diversos investigadores detectaron su gran influencia en países industrializados. Gracias a ello dejó de contemplarse como un vestigio del pasado, pues formaba parte de nuevas expectativas y esperanzas de crecimiento económico, oportunidad de empleos y estrategia de competitividad empresarial. Ante ello, se dejó de pensar que este sector se encontraba en una etapa de paulatina desaparición y se empezó a interpretar como parte de las primeras formas de desarrollo capitalista (Saraví, 1997). 


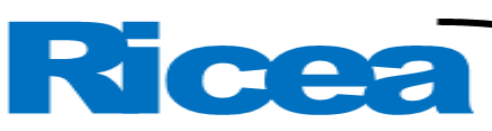

El concepto microempresa se refiere a la unidad económica involucrada en actividades relacionadas con la industria manufacturera, el comercio, los servicios, la construcción y el transporte. Estas actividades las puede realizar en la vivienda o fuera de ella con o sin local, y ocupar entre 1 y 10 empleados.

Además, toda organización empresarial denominada microempresa debe reunir las siguientes características (Rodríguez, 2000):

Propietarios y administración independiente.

Excepción de las familiares.

No domina el sector de la actividad donde opera.

Estructura organizacional muy sencilla.

Sus ventas anuales no sobrepasen los 30 millones de pesos.

Ahora bien, para cualquier investigación también resulta esencial analizar el origen de su objeto de estudio. En este caso, esa tarea puede resultar compleja porque esta unidad económica puede compartir los mismos espacios económicos de la pequeña, mediana y gran empresa, ya que en aquella también se presentan la asociación, la cooperación empresarial, el intercambio de servicios y productos, incluso como parte de estructuras de mercado. Por ello, en esta investigación se procura contextualizar la diversidad de criterios que sustentan la división de empresas por tamaños.

Hasta fines de los años setenta y con motivos del fomento y estudio de la subdivisión de las empresas, solo existían tres estratos: pequeña, mediana y gran empresa. Sin embargo, a mitad de los años ochenta - y como resultado de una gran crisis económica y de los grandes conflictos políticos a nivel internacional que dificultaban el empleo en sectores urbanos de menor poder económico- nació el modelo de microempresa. Esta nueva figura permitía a las personas obtener ingresos para asegurar las necesidades básicas. Esta unidad económica se potenció en la década de los noventa, cuando las políticas económicas fueron desacertadas y presentaron serios desequilibrios en el desarrollo, lo que originó la quiebra de la mediana y pequeña empresa, así como el consiguiente desempleo y endeudamiento del sector privado.

Para México la situación no fue sencilla: las pequeñas empresas con pasivos sorpresivamente duplicaron sus deudas y la oportunidad de subsanar sus problemas se convirtió en un reto. No existían activadores económicos o programas que realmente los impulsaran; el ingenio y el talento de muchos mexicanos se pusieron a prueba y encontraron una solución a su situación económica mediante el surgimiento de miles de microempresas y pequeños negocios familiares que tenían un promedio inferior a diez 


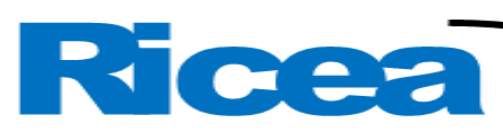

- Poca o ninguna especialización en la administración. Se considera esta característica derivada directamente de la naturaleza de dichas empresas, cuya constitución obedece a motivos económicos y de subsistencia de los dueños. En otras palabras, ellos suelen dirigir la empresa de manera empírica, pues carecen de capacitación para desempeñar esos cargos.

- Poco conocimiento de los dueños sobre posibles fuentes de financiamiento. Este es un problema generalizado en este tipo de empresas debido a que los dueños desconocen esa información.

- Dependencia total de la comunidad y su entorno. La pequeña empresa depende en su mayor parte de la situación económica de la comunidad en donde se localiza; por ello, su economía es el reflejo de su entorno, del cual necesita para obtener bienes de producción, mano de obra y clientes. En este sentido, son pocas las que han rebasado el límite local o regional, y mucho menos las que se dedican a la exportación.

Además de lo señalado, existen severas confusiones entre los diversos tipos de empresas desde las esferas gubernamentales de cualquier nivel. En un estudio realizado por el Instituto de Investigaciones Legislativas del Senado de la República se reconoce que en México ha sido difícil ubicarlas correctamente a través del tiempo. Esta situación surge porque las variables e indicadores que se han tomado en cuenta para clasificarlas han cambiado constantemente. Además, de manera tradicional, se han utilizado parámetros económicos y contables para clasificarlas, tales como el número de trabajadores, el total de ventas anuales, los ingresos y los activos fijos de las empresas. Al respecto, Cuitlahuac Bardan Esquivel (2002) señala:

En abril de 1954 la Secretaría de Hacienda consideró como pequeños y medianos industriales aquellos cuyo capital contable no fuera menor de 50000 y tampoco mayor de 2500000 pesos. Para 1961 se tomó en cuenta un capital contable con mayor margen: desde 25 000, hasta 5 millones de pesos para clasificar a las pequeñas y medianas industrias. Y en 1963, la Secretaría de Hacienda modificó las reglas de operación y comenzó a considerar como pequeñas y medianas empresas a las que tuvieran un capital contable no mayor de 10 millones de pesos (párr. 6).

Esta variabilidad en las determinaciones sitúa a las microempresas fuera del entorno empresarial, pues solo se distinguen a aquellas que tienen una organización, una estructura, una gestión empresarial y el trabajo remunerado. En otras palabras, las de 


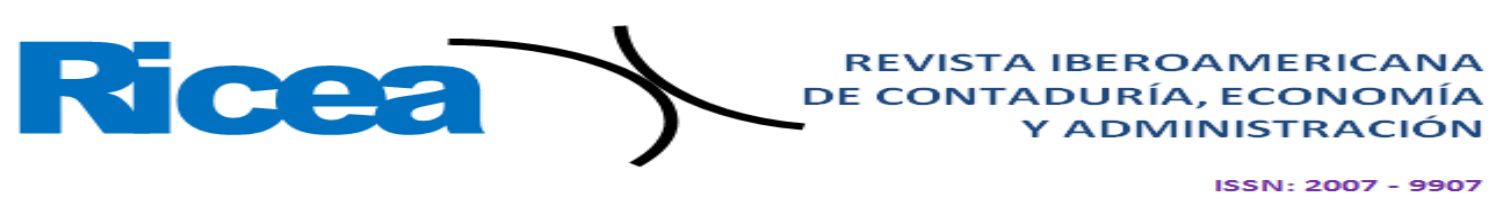

- Datos generales del negocio claramente expuestos en el lugar: dirección, giro del negocio, datos de contacto.

- Consideración del tema antes de la apertura: si el emprendedor o propietario del negocio consideró el tema como parte elemental de su empresa.

- Si conoce el concepto de fuerza de ventas, y su aplicación desde la apertura.

- Manejo de redes sociales o medios digitales para dar a conocer a la microempresa comercial.

- Implementar estrategias planificadas de ventas desde su apertura.

Con estos puntos como referencia se procedió a realizar el levantamiento de encuestas con un instrumento previamente avalado por los expertos participantes. Gracias a la Cámara de Comercio local, se identificaron las microempresas comerciales que cubrían el requisito de 5 años o más en funciones. En este proceso se realizó un comparativo de registros en la misma Cámara, y se detectaron 87 microempresas comerciales que cerraron por diferentes razones, las cuales tuvieron un periodo de vida no mayor de 3 años. De estas microempresas, algunos propietarios cambiaron de domicilio, dejaron de vivir en el estado o los datos de contacto no coincidieron. Se logró establecer comunicación con 35 de ellos, de los cuales accedieron a una entrevista solamente 27 microempresarios.

Las microempresas que cerraron no lo hicieron exclusivamente debido a la falta de estrategias mercadológicas. En este sentido, se debe reconocer que existen muchos factores que pueden desencadenar esta decisión. Aun así, intentamos conocer si alguna estrategia mercadológica hubiera contribuido a evitar el cierre.

Entre las causas más frecuentes encontradas en los 27 microempresarios consultados, $54 \%$ expresaron que el pago de la renta fue el principal obstáculo, $8 \%$ mencionó la falta de clientes o mercado, $6 \%$ falta de recursos económicos, $19 \%$ pocas ventas, $5 \%$ dificultad de empleados honestos, $4 \%$ por encontrar un empleo, $3 \%$ por inseguridad y violencia y $1 \%$ por motivos familiares.

Una vez detectadas las deficiencias que los propietarios manifestaron al cerrar su microempresa comercial, se les informó de las ventajas que ofrecen las acciones mercadológicas para los negocios. Se les preguntó si llegaron a aplicar, en algún momento de su permanencia, algunas de estas estrategias. Se obtuvieron las respuestas siguientes: $75 \%$ de los entrevistados no dieron importancia a la asignación de un nombre para su negocio, $67 \%$ reconoció que incluso no eligieron un nombre o negaron hacerlo público en el establecimiento. $83 \%$ mencionaron el nulo conocimiento acerca de una identidad 


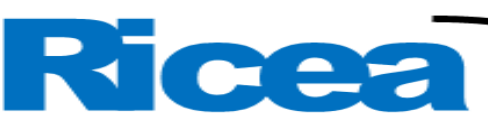

REVISTA IBEROAMERICANA DE CONTADURÍA, ECONOMÍA Y ADMINISTRACIÓN

La expectativa del género en un microempresario respecto a su sobrevivencia determina que las microempresas comerciales femeninas son más susceptibles al cierre que las masculinas. Con el análisis de las microempresas cerradas el fenómeno ocurre de la misma manera, pues $64.8 \%$ eran de emprendimiento femenino. Se registró un nivel de significancia de $1 \%$, aplicando la $\mathrm{x} 2$ de Pearson. Por tanto, se cumple la $\mathrm{H} 2$, respecto a la influencia del género en la consolidación de las empresas.

Tabla 3. Género del microempresario

\begin{tabular}{|c|c|c|c|}
\hline Rango & Cierre & Mercadotecnia & Sig. \\
\hline Femenino & 52.7 & 64.8 & \\
\hline Masculino & 47.3 & 35.2 & $* * *$ \\
\hline Total & $100 \%$ & $100 \%$ & \\
\hline \multicolumn{4}{|c|}{ Chi cuadrado de Pearson: 26.55/ gl:2/sig. Asintótica (bilateral): 0.000} \\
\hline
\end{tabular}

Fuente Elaboración propia

En cuanto a si el nivel educativo incide en el fracaso o cierre de las microempresas comerciales, es válida solamente con una significancia de $5 \%$, el menor nivel educativo eleva la probabilidad de cierre o fracaso en la microempresa comercial y no se pueda consolidar. Con esto se valida la $\mathrm{H} 3$, que señala que a mayor conocimiento el fracaso de la microempresa es menor.

Tabla 4. Educación del microempresario

\begin{tabular}{|c|c|c|c|}
\hline Nivel Educativo & Cierre & Mercadotecnia & Sig. \\
\hline $\begin{array}{l}\text { Sin educación } \\
\text { formal }\end{array}$ & 9.6 & 5.3 & \\
\hline $\begin{array}{l}\text { Educación básica } \\
\text { hasta bachillerato }\end{array}$ & 72.8 & 70.6 & $* * *$ \\
\hline Educación superior & 17.6 & 24.1 & \\
\hline Total & $100 \%$ & $100 \%$ & \\
\hline \multicolumn{4}{|c|}{$\begin{array}{l}\text { Chi Cuadrado de Pearson: 9.98/ gl:4/sig. Asintótica (bilateral): } 0.041 \\
(*): \mathrm{p}<0.1 ;(* *): \mathrm{p}<0.05 ;(* * *): \mathrm{p} 0.01 . \mathrm{N}=1036\end{array}$} \\
\hline
\end{tabular}

Fuente Elaboración propia 


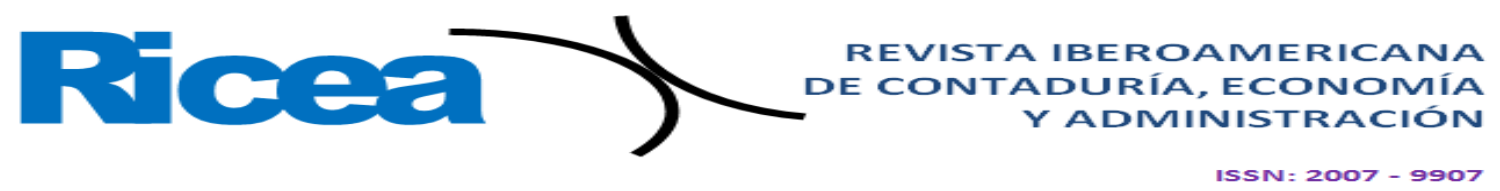

de https://www.researchgate.net/profile/Rachida_Justo/publication/23528730_G ender_family_situation_and_the_exit_event_reassessing_the_opportunitycosts_of_business_ownership/links/0c96052527a4ac9364000000/Genderfamily-situation-and-the-exit-event-reassessing-the-opportunity-costs-ofbusiness-ownership.pdf?origin=publication_detail

Lévesque, M. y Minniti, M. (2003). The effect of aging on entrepreneurial behavior. Technical Memorandum Number 774. Recuperado el 20/06/2012 de http://weatherhead.case.edu/departments/operations/research/technicalReports/T echnical\%20Memorandum\%20Number\%20774.pdf.

Méndez, C. (2011) Diagnóstico de la MYPIMES en México, aportación del Sistema de Información Empresarial de México. México, D.F.

Montes-Rojas, G. y Siga, L. (2009).On the nature of micro-entrepreneurship: evidence from Argentina. Applied Economics, 41(21), (pp. 2667-2680). Recuperado 06/10/2019 de http://econ.ucsd.edu/ 1siga/pdfs/Entrepr_Arg.pdf.

Navarrete, E. y Sansores, E. (2011). "El fracaso de las micro, pequeñas y medianas empresas en Quintana Roo, México: un análisis multivariante”. Revista Internacional de Administración y Finanzas, 21-33.

Powers, J. y Magnoni, B. (2010). Dueña de tu propia empresa: Identificación, análisis y superación de las limitaciones a las pequeñas empresas de las mujeres en América Latina y el Caribe. Washington, D.C.: Fondo Multilateral de Inversiones, BID

Rodríguez, J. (2000). Administración de pequeñas y medianas empresas. México, D.F. Editorial Thomson.

Saraví, G. (1997). Redescubriendo la microindustria. Dinámica y configuración de un distrito industrial en México. Chicago Illinois: Juan Pablo Editor.

Woo, Cooper . and Dunkelberg (1994). Small Business: Critical Perspectives on Business and Management. Journal of Business Venturing, 4, 317-332. 


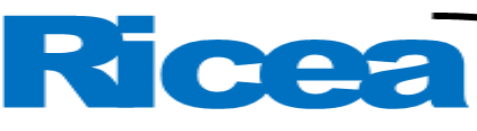

\begin{tabular}{|c|c|}
\hline Rol de Contribución & Autor (es) \\
\hline Conceptualización & $\begin{array}{l}\text { Raúl Alonso Lozada- Principal } \\
\text { Armando Villanueva Meléndez -igual } \\
\text { Adriana Montiel García -apoyo }\end{array}$ \\
\hline Metodología & $\begin{array}{l}\text { Raúl Alonso Lozada- Principal } \\
\text { Armando Villanueva Meléndez -igual } \\
\text { Adriana Montiel García -apoyo } \\
\text { Ma. Luisa Espinoza Aguila -apoyo }\end{array}$ \\
\hline Software & $\begin{array}{l}\text { Armando Villanueva Meléndez -Principal } \\
\text { Ma. Luisa Espinoza Aguila -apoyo } \\
\text { Julissa Tizapantzi Sánchez -apoyo }\end{array}$ \\
\hline Validación & $\begin{array}{l}\text { Raúl Alonso Lozada-Principal } \\
\text { Ma. Luisa Espinoza Aguila -apoyo }\end{array}$ \\
\hline Análisis Formal & $\begin{array}{l}\text { Raúl Alonso Lozada-Principal } \\
\text { Armando Villanueva Meléndez -igual } \\
\text { Adriana Montiel García -apoyo }\end{array}$ \\
\hline Investigación & $\begin{array}{l}\text { Raúl Alonso Lozada- Principal } \\
\text { Armando Villanueva Meléndez -igual } \\
\text { Adriana Montiel García -apoyo } \\
\text { Ma. Luisa Espinoza Aguila -apoyo }\end{array}$ \\
\hline Recursos & $\begin{array}{l}\text { Ma. Luisa Espinoza Aguila -Principal } \\
\text { Julissa Tizapantzi Sánchez -Apoyo }\end{array}$ \\
\hline Curación de datos & Julissa Tizapantzi Sánchez -Principal \\
\hline $\begin{array}{l}\text { Escritura - Preparación } \\
\text { del } \\
\text { borrador original }\end{array}$ & $\begin{array}{l}\text { Armando Villanueva Meléndez -Principal } \\
\text { Raúl Alonso Lozada -igual } \\
\text { Ma. Luisa Espinoza Aguila -apoyo }\end{array}$ \\
\hline $\begin{array}{l}\text { Escritura - Revisión } \quad \text { y } \\
\text { Edición }\end{array}$ & $\begin{array}{l}\text { Raúl Alonso Lozada -Principal } \\
\text { Adriana Montiel García -apoyo } \\
\text { Ma. Luisa Espinoza Aguila -apoyo }\end{array}$ \\
\hline Visualización & Julissa Tizapantzi Sánchez -Principal \\
\hline Supervisión & $\begin{array}{l}\text { Armando Villanueva Meléndez -Principal } \\
\text { Raúl Alonso Lozada -igual }\end{array}$ \\
\hline $\begin{array}{l}\text { Administración de } \\
\text { Proyectos }\end{array}$ & Julissa Tizapantzi Sánchez -Principal \\
\hline Adquisición de fondos & Armando Villanueva Meléndez -Principal \\
\hline
\end{tabular}

